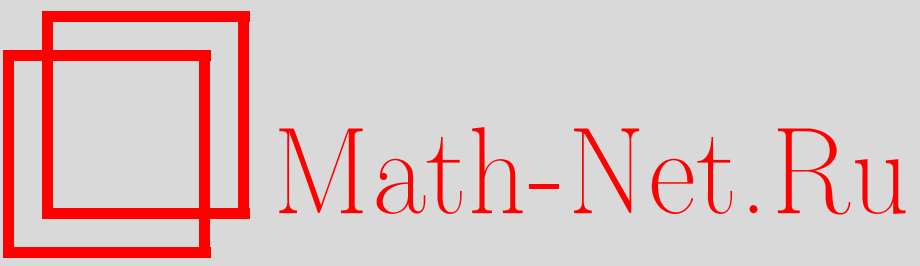

П. М. Симонов, Устойчивость и асимптотически периодические решения гибридных систем с последействием, Итоги науки и техн. Сер. Соврем. мат. и ее прил. Темат. обз., 2019, том 168, 91-98

DOI: https://doi.org/10.36535/0233-6723-2019-168-91-98

Использование Общероссийского математического портала Math-Net.Ru подразумевает, что вы прочитали и согласны с пользовательским соглашением

http://www.mathnet.ru/rus/agreement

Параметры загрузки:

IP: 54.209 .52 .79

26 апреля 2023 г., 14:17:07 


\title{
УСТОЙЧИВОСТЬ И АСИМПТОТИЧЕСКИ ПЕРИОДИЧЕСКИЕ РЕШЕНИЯ ГИБРИДНЫХ СИСТЕМ С ПОСЛЕДЕЙСТВИЕМ
}

\author{
(c) 2019 г. $\quad$ П. М. СИМОнов
}

\begin{abstract}
АннотАция. В статье продолжается изучение гибридной линейной системы функциональнодифференциальных уравнений с последействием при помощи $W$-метода Н. В. Азбелева. Рассмотрены два модельных уравнения, изучены банаховы пространства правых частей и решений, являющиеся пространствами асимптотически периодических функций. Получены аналоги теоремы Боля-Перрона об асимптотической устойчивости и о существовании пределов решений.
\end{abstract}

Ключевые слова: теорема Боля-Перрона, асимптотически периодические функции, гибридная система, функционально-дифференциальное уравнение, уравнение с последействием, устойчивость, метод модельных уравнений.

\section{STABILITY AND ASYMPTOTICALLY PERIODIC SOLUTIONS OF HYBRID SYSTEMS WITH AFTEREFFECT}

\author{
(C) 2019 P. M. SIMONOV
}

\begin{abstract}
In this paper, we study hybrid linear systems of functional differential equations with aftereffect using the Azbelev $W$-method. Two model equations are considered and Banach spaces of right-hand sides and solutions, which are spaces of asymptotically periodic functions, are examined. Analogs of the Bohl-Perron theorem on the asymptotic stability and on the existence of limits of solutions are obtained.
\end{abstract}

Keywords and phrases: Bohl-Perron theorem, asymptotically periodic functions, hybrid system, functional differential equation, equation with aftereffect, stability, method of model equations.

AMS Subject Classification: $34 \mathrm{~K} 20,34 \mathrm{~K} 25$

1. Введение. В настоящей статье мы продолжаем изучение гибридных линейных систем функционально-дифференциальных уравнений с последействием (ГЛСФДУП). Гибридность понимается в следующем смысле: система является системой линейных функционально-дифференциальных уравнений с последействием (ЛФДУП) по одной части переменных и системой линейных разностных уравнений с последействием (ЛРУП) по другой части переменных. Применяя $W$-метод Н. В. Азбелева, мы изучаем два модельных уравнения и банаховы пространства их правых частей и решений (пространства асимптотически периодических функций). Получены аналоги теоремы Боля-Перрона об асимптотической устойчивости и о существовании пределов решений, доказанной ранее в работах Н. В. Азбелева, Л. М. Березанского, П. М. Симонова и А. В. Чистякова (см. [1-4]).

Рассмотрим пространство $\mathbb{R}^{n}$ векторов $\alpha=\operatorname{col}\left\{\alpha^{1}, \ldots, \alpha^{n}\right\}$ с действительными компонентами и с нормой $\|\alpha\|_{\mathbb{R}^{n}}$.

Работа выполнена при поддержке Российского фонда фундаментальных исследований (проект № 18-01-00332-а). 
Обозначим через $L$ пространство локально суммируемых функций $f:[0, \infty) \rightarrow \mathbb{R}^{n}$ с полунормами

$$
\|f\|_{L[0, T]}=\int_{0}^{T}\|f(t)\|_{\mathbb{R}^{n}} d t, \quad T>0,
$$

и через $D$ - пространство локально абсолютно непрерывных функций $x:[0, \infty) \rightarrow \mathbb{R}^{n}$ с полунормами

$$
\|x\|_{D[0, T]}=\|\dot{x}\|_{L[0, T]}+\|x(0)\|_{\mathbb{R}^{n}}, \quad T>0 .
$$

Обозначим через $y=\{y(-1), y(0), y(1), \ldots, y(N), \ldots\}$ бесконечную матрицу со столбцами $y(-1), y(0), y(1), \ldots, y(N), \ldots$ высоты $n$, а через $g=\{g(0), g(1), \ldots, g(N), \ldots\}$ бесконечную матрицу со столбцами $g(0), g(1), \ldots, g(N), \ldots$ высоты $n$. Каждой бесконечной матрице $y=$ $\{y(-1), y(0), y(1), \ldots, y(N), \ldots\}$ можно поставить в соответствие вектор-функцию

$$
y(t)=y(-1) \chi_{[-1,0)}(t)+y(0) \chi_{[0,1)}(t)+y(1) \chi_{[1,2)}(t)+\cdots+y(N) \chi_{[N, N+1)}(t)+\ldots
$$

Аналогично, каждой бесконечной матрице $g=\{g(0), g(1), \ldots, g(N), \ldots\}$ можно поставить в соответствие вектор-функцию

$$
g(t)=g(0) \chi_{[0,1)}(t)+g(1) \chi_{[1,2)}(t)+\cdots+g(N) \chi_{[N, N+1)}(t)+\ldots
$$

Обозначим через $y(t)=y[t]$ вектор-функцию $y(t)=y([t]), t \in[-1, \infty)$, через $g[t]$ - вектор-функцию $g(t)=g([t]), t \in[0, \infty)$, через $\ell_{0}$ - множество вектор-функций $y[\cdot]$ с полунормами

$$
\|y\|_{\ell_{0 T}}=\sum_{i=-1}^{T}\left\|y_{i}\right\|_{\mathbb{R}^{n}}, \quad T \geqslant-1,
$$

через $\ell$ - множество вектор-функций $g[\cdot]$ с полунормами

$$
\|g\|_{\ell_{T}}=\sum_{i=0}^{T}\left\|g_{i}\right\|_{\mathbb{R}^{n}}, \quad T \geqslant 0 .
$$

Введем также обозначения

$$
\begin{array}{ll}
(\Delta y)(t)=y(t)-y(t-1)=y[t]-y[t-1] & \text { при } t \geqslant 1, \\
(\Delta y)(t)=y(t)=y[t]=y(0) & \text { при } t \in[0,1) .
\end{array}
$$

Запишем абстрактную гибридную функционально-дифференциальную систему в виде

$$
\begin{aligned}
& \mathcal{L}_{11} x+\mathcal{L}_{12} y=\dot{x}-F_{11} x-F_{12} y=f, \\
& \mathcal{L}_{21} x+\mathcal{L}_{22} y=\Delta y-F_{21} x-F_{22} y=g .
\end{aligned}
$$

Операторы $\mathcal{L}_{11}, F_{11}: D \rightarrow L, \mathcal{L}_{12}, F_{12}: \ell_{0} \rightarrow L, \mathcal{L}_{21}, F_{21}: D \rightarrow \ell, \mathcal{L}_{22}, F_{22}: \ell_{0} \rightarrow \ell$ предполагаются линейными непрерывными и вольтерровыми.

Если элементы $\operatorname{col}\{x, y\}:[0, \infty) \times[-1, \infty) \rightarrow \mathbb{R}^{n} \times \mathbb{R}^{n}$, образующие банахово пространство

$$
\boldsymbol{D} \times \boldsymbol{M}_{0} \cong\left(\boldsymbol{B} \times \mathbb{R}^{n}\right) \times\left(\boldsymbol{M} \times \mathbb{R}^{n}\right),
$$

где $\boldsymbol{D} \subset D, \boldsymbol{M}_{0} \subset \ell_{0}, \boldsymbol{B} \subset L, \boldsymbol{M} \subset \boldsymbol{\ell}(\boldsymbol{B}, \boldsymbol{M}$-банаховы пространства), обладают какими-либо специфическими свойствами, например

$$
\sup _{t \geqslant 0}\|x(t)\|_{\mathbb{R}^{n}}+\sup _{k=-1,0,1, \ldots}\|y(k)\|_{\mathbb{R}^{n}}<\infty,
$$

и для уравнения $\mathcal{L}\{x, y\}=\operatorname{col}\{f, g\}$, где $\mathcal{L}: \boldsymbol{D} \times \boldsymbol{M}_{0} \rightarrow \boldsymbol{B} \times \boldsymbol{M}$-линейный ограниченный оператор, однозначно разрешима задача Коши, то и решения этой задачи будут обладать такими же асимптотическими свойствами.

Пусть модельное уравнение $\mathcal{L}_{11} x=z$ (см. [1-4]) и банахово пространство $\boldsymbol{B} \subset L$ (вложение непрерывно) выбраны так, что решения этого уравнения обладают интересующими нас асимптотическими свойствами. Пусть при любом $z \in \boldsymbol{B}$ решение записывается в виде формулы Коши

$$
x(t)=U_{11}(t) x(0)+\left(W_{11} z\right)(t) .
$$


Для банахова пространства $\boldsymbol{B} \subset L$ можно ввести банахово пространство $\boldsymbol{D}\left(\mathcal{L}_{11}, \boldsymbol{B}\right)$ с нормой

$$
\|x\|_{\boldsymbol{D}\left(\mathcal{L}_{11}, \boldsymbol{B}\right)}=\left\|\mathcal{L}_{11} x\right\|_{\boldsymbol{B}}+\|x(0)\|_{\mathbb{R}^{n}} .
$$

Здесь вложение $\boldsymbol{B} \subset L$ непрерывно. Предположим, что оператор $W_{11}$ непрерывно действует из пространства $\boldsymbol{B}$ в пространство $\boldsymbol{B}$, а оператор $U_{11}$ действует из пространства $\mathbb{R}^{n}$ в пространство $\boldsymbol{B}$. Это условие эквивалентно тому (см. [3], [4, гл. IV]), что пространство $\boldsymbol{D}\left(\mathcal{L}_{11}, \boldsymbol{B}\right)$ линейно изоморфно пространству Соболева $W_{\boldsymbol{B}}^{(1)}[0, \infty)$ с нормой

$$
\|x\|_{W_{B}^{(1)}[0, \infty)}=\|\dot{x}\|_{B}+\|x\|_{B} ;
$$

в дальнейшем будем обозначать это пространство $\boldsymbol{W}_{\boldsymbol{B}}$; при этом $\boldsymbol{W}_{\boldsymbol{B}} \subset D$ (вложение непрерывно).

Будем говорить, что уравнение $\mathcal{L}_{11} x=z$ с оператором $\mathcal{L}_{11}: \boldsymbol{D}\left(\mathcal{L}_{11}\right) \rightarrow \boldsymbol{B}$ является $\boldsymbol{D}\left(\mathcal{L}_{11}, \boldsymbol{B}\right)$ устойчивым, если для каждой правой части $z \in \boldsymbol{B}$ решение $x$ принадлежит $\boldsymbol{D}\left(\mathcal{L}_{11}, \boldsymbol{B}\right)$ (см. [1]); здесь $\boldsymbol{D}\left(\mathcal{L}_{11}\right) \subset D$ - область определения оператора $\mathcal{L}_{11}$. Уравнение $\mathcal{L}_{11} x=z$ называется сильно $\boldsymbol{B}$-устойчивым, если для любого $z \in \boldsymbol{B}$ каждое решение $x$ этого уравнения обладает следующим свойством: $x \in \boldsymbol{B}$ и $\dot{x} \in \boldsymbol{B}$ (см. [3], [4, гл. IV, $\S 4.6]$ ). Уравнение $\mathcal{L}_{11} x=z$ с оператором $\mathcal{L}_{11}: \boldsymbol{D}\left(\mathcal{L}_{11}, \boldsymbol{B}\right) \rightarrow \boldsymbol{B}$, удовлетворяющим приведенному выше условию, является $\boldsymbol{D}\left(\mathcal{L}_{11}, \boldsymbol{B}\right)$-устойчивым тогда и только тогда, если оно сильно $\boldsymbol{B}$-устойчиво.

Операторы $\mathcal{L}_{11}: D \rightarrow L, \mathcal{L}_{12}: \ell_{0} \rightarrow L, \mathcal{L}_{21}: D \rightarrow \ell, \mathcal{L}_{22}: \ell_{0} \rightarrow \ell$ рассматриваются как приведения на пары $\left(\boldsymbol{D}\left(\mathcal{L}_{11}^{0}, \boldsymbol{B}\right), \boldsymbol{B}\right),\left(\boldsymbol{M}_{0}, \boldsymbol{B}\right),\left(\boldsymbol{D}\left(\mathcal{L}_{11}^{0}, \boldsymbol{B}\right), \boldsymbol{M}\right),\left(\boldsymbol{M}_{0}, \boldsymbol{M}\right)$; здесь $\boldsymbol{W}_{\boldsymbol{B}}=\boldsymbol{D}=$ $\boldsymbol{D}\left(\mathcal{L}_{11}^{0}, \boldsymbol{B}\right)$. Эти операторы предполагаются линейными, вольтерровыми и ограниченными.

Введем следующие пространства:

$$
\begin{aligned}
\ell_{\infty 0} & =\left\{y \in \ell_{0}:\|y\|_{\ell_{\infty} 0}=\sup _{k=-1,0,1, \ldots}\|y(k)\|_{\mathbb{R}^{n}}<+\infty\right\}, \\
\ell_{\infty} & =\left\{g \in \ell:\|g\|_{\ell_{\infty}}=\sup _{k=0,1, \ldots}\|g(k)\|_{\mathbb{R}^{n}}<+\infty\right\} .
\end{aligned}
$$

Банаховы пространства $\ell_{\infty 0}$ и $\ell_{\infty}$ являются примерами пространств типа $\boldsymbol{M}_{0}$ и $\boldsymbol{M}$.

Введем обозначение

$$
\mathcal{L}=\left(\begin{array}{ll}
\mathcal{L}_{11} & \mathcal{L}_{12} \\
\mathcal{L}_{21} & \mathcal{L}_{22}
\end{array}\right)
$$

Тогда уравнение (1) записывается в виде

$$
\mathcal{L}\{x, y\}=\operatorname{col}\{f, g\} .
$$

Предположим, что для любых $x(0) \in \mathbb{R}^{n}$ и $y(-1) \in \mathbb{R}^{n}$ задача Коши для «модельной» системы

$$
\begin{aligned}
& \mathcal{L}_{11}^{0} x+\mathcal{L}_{12}^{0} y=\dot{x}-F_{11}^{0} x-F_{12}^{0} y=z \\
& \mathcal{L}_{21}^{0} x+\mathcal{L}_{22}^{0} y=\Delta y-F_{21}^{0} x-F_{22}^{0} y=u
\end{aligned}
$$

однозначно разрешима; здесь операторы $F_{11}^{0}: D \rightarrow L, F_{12}^{0}: \ell_{0} \rightarrow L, F_{21}^{0}: D \rightarrow \ell, F_{22}^{0}: \ell_{0} \rightarrow \ell$ предполагаются непрерывными и вольтерровыми. Тогда модельную систему можно коротко записать в виде

$$
\mathcal{L}_{0}\{x, y\}=\operatorname{col}\{z, u\} .
$$

Пусть ее решение имеет представление

$$
\left(\begin{array}{l}
x \\
y
\end{array}\right)=\left(\begin{array}{ll}
U_{11} & U_{12} \\
U_{21} & U_{22}
\end{array}\right)\left(\begin{array}{c}
x(0) \\
y(-1)
\end{array}\right)+\left(\begin{array}{ll}
W_{11} & W_{12} \\
W_{21} & W_{22}
\end{array}\right)\left(\begin{array}{l}
z \\
u
\end{array}\right)
$$

через $\mathcal{U}: \mathbb{R}^{n} \times \mathbb{R}^{n} \rightarrow D \times \ell_{0}$ и $\mathcal{W}: L \times \ell \rightarrow D \times \ell_{0}$ обозначим соответственно фундаментальную матрицу и непрерывный вольтерров оператор Коши системы:

$$
\mathcal{U}=\left(\begin{array}{ll}
U_{11} & U_{12} \\
U_{21} & U_{22}
\end{array}\right), \quad \mathcal{W}=\left(\begin{array}{ll}
W_{11} & W_{12} \\
W_{21} & W_{22}
\end{array}\right)
$$


Предположим, что общее решение уравнения $\mathcal{L}_{22} y=g$ для $g \in \boldsymbol{M}$ принадлежит пространству $\boldsymbol{M}_{0}$ и представляется формулой Коши:

$$
y[t]=\left(\mathcal{Y}_{11} y(-1)\right)[t]+\left(\mathcal{C}_{22} g\right)[t]=Y_{22}[t] y(-1)+\sum_{s=0}^{t} C_{22}[t, s] g[s], \quad t \geqslant 0 .
$$

2. Теоремы Боля-Перрона. Для обыкновенного дифференциального уравнения еще в монографиях $[5,6,10,11]$ отмечались явления, которые в терминах $\boldsymbol{D}\left(\mathcal{L}_{11}, \boldsymbol{B}\right)$-устойчивости можно сформулировать следующим образом. При определенных условиях относительно оператора $\mathcal{L}_{11}$ из $\boldsymbol{D}\left(\mathcal{L}_{11}, \boldsymbol{B}\right)$-устойчивости следует более тонкое асимптотическое свойство, а именно $\boldsymbol{D}\left(\mathcal{L}_{11}, \boldsymbol{B}_{1}\right)$ устойчивость, где $\boldsymbol{B}_{1}$ - некоторое подпространство пространства $\boldsymbol{B}$.

Следуя традиции Пермского семинара (см. [1-4]), соответствующие утверждения будем называть теоремами Боля-Перрона. В основе доказательств таких теорем, приведенных ниже, лежат свойства подпространства $\boldsymbol{B} \subset L$, вытекающие из их порядковой структуры, которую определим следующим образом. В векторном пространстве $\mathbb{R}^{n}$ введем частичную упорядоченностъ: $\alpha=\operatorname{col}\left\{\alpha_{1}, \ldots, \alpha_{n}\right\} \geqslant 0$, если $\alpha_{i} \geqslant 0, i=1, \ldots, n ;$ соответственно, $\alpha \geqslant \beta$, если $\alpha-\beta \geqslant 0$. Через $|\alpha|$ будем обозначать вектор, определяемый равенством $|\alpha|=\operatorname{col}\left\{\left|\alpha_{1}\right|, \ldots,\left|\alpha_{n}\right|\right\}$. Будем предполагать, что в пространстве $\mathbb{R}^{n}$ зафиксирована норма $\|\cdot\|_{\mathbb{R}^{n}}$, обладающая свойством монотонности:

$$
\|\alpha\|_{\mathbb{R}^{n}} \leqslant\|\beta\|_{\mathbb{R}^{n}}, \quad \text { если }|\alpha| \leqslant|\beta| .
$$

В соответствии с порядком в пространстве $\mathbb{R}^{n}$ введем отношение порядка в пространстве $L$ : будем считать, что $y \geqslant 0$, если $y(t) \geqslant 0$ почти всюду на $[0, \infty) ; y \geqslant z$, если $y-z \geqslant 0$. Через $|y|$ будем обозначать функцию, почти всюду на $[0, \infty)$ определяемую равенством $|y|(t)=|y(t)|$. Относительно банахова пространства $\boldsymbol{B} \subset L$ будем предполагать, что норма в пространстве $\boldsymbol{B}$ согласована с порядком через условие идеальности: если $z \in L, y \in \boldsymbol{B}$ и $|z| \leqslant|y|$, то $z \in \boldsymbol{B}$ и $\|z\|_{B} \leqslant\|y\|_{B}$.

Среди прочих свойств пространств, удовлетворяющих этому условию (банаховых идеалъных пространств; см. [9][гл. IV, § 3, п. 3.1; гл. Х, § 4, п. 4.1]), отметим следующие:

(1) норма в таком пространстве $\boldsymbol{B}$ обладает свойством монотонности;

(2) любое ограниченное по порядку подмножество пространства $\boldsymbol{B}$ имеет точные грани $(\boldsymbol{B}-K$ -пространство);

(3) в пространстве $\boldsymbol{B}$ определены «срезки»-операторы умножения на характеристические функции $\chi_{M}$ измеримого множества $M \subset[0, \infty)$;

(4) вложение $\boldsymbol{B} \subset L$ непрерывно.

3. Асимптотическая устойчивость. Введем подмножество $\boldsymbol{B}_{0} \subset \boldsymbol{B}$ всех функций $z \in \boldsymbol{B}$, удовлетворяющих условию

$$
\lim _{s \rightarrow \infty}\left\|\chi_{[s, \infty)} z\right\|_{\boldsymbol{B}}=\lim _{s \rightarrow \infty}\left\|z^{s}\right\|_{\boldsymbol{B}}=0 .
$$

Здесь и ниже $\chi_{M}-$ характеристическая функция множества $M$. Иначе говоря, пространство $\boldsymbol{B}_{0}$ состоит из всех функций $z \in \boldsymbol{B}$, стремящихся при $t \rightarrow \infty$ к нулю по метрике пространства $\boldsymbol{B}$. Нетрудно показать, что $\boldsymbol{B}_{0}$ является замкнутым подпространством пространства $\boldsymbol{B}$ и совпадает с замыканием по норме $\|\cdot\|_{\boldsymbol{B}}$ линейного многообразия всех финитных функций $z \in \boldsymbol{B}$. Далее, пусть $C_{0}$ - подпространство пространства $C$, состоящее из всех $x \in C$, для которых $\lim _{t \rightarrow \infty} x(t)=$ $0,\|x\|_{C_{0}}=\|x\|_{C}$. Здесь и ниже $C$-пространство непрерывных и ограниченных функций $x:$ $[0, \infty) \rightarrow \mathbb{R}^{n}$ с нормой $\|x\|_{C}=\sup _{t \geqslant 0}\|x(t)\|_{\mathbb{R}^{n}}$.

Введем обозначение $Q_{11}=\mathcal{L}_{11} W_{11}$. Введем банаховы пространства всех функций $g \in \ell_{\infty}^{0} \subset \ell_{\infty}$ (соответственно, $g \in \ell_{\infty 0}^{0} \subset \ell_{\infty 0}$ ), удовлетворяющих условиям

$$
\lim _{n \rightarrow \infty}\left\|\chi_{[n, n+1, \cdots, \infty)} g\right\|_{\ell_{\infty}}=0 \quad\left(\lim _{n \rightarrow \infty}\left\|\chi_{[n, n+1, \cdots, \infty)} g\right\|_{\ell_{\infty}}=0\right) .
$$

Рассмотрим банахово пространство $\boldsymbol{b} \subset \ell$ бесконечных матриц $g=\{g(0), g(1), \ldots, g(N), \ldots\}$ с нормой $\|g\|_{\boldsymbol{b}}$ и банахово пространство $\boldsymbol{b}_{0} \subset \ell_{0}$ бесконечных матриц $y=\{y(-1), y(0), y(1), \ldots, y(N), \ldots\}$ 
с нормой $\|y\|_{\boldsymbol{b}_{0}}$, причем вложения $\boldsymbol{b} \subset \ell$ и $\boldsymbol{b}_{0} \subset \ell_{0}$ непрерывны. Предположим, что в банаховом пространстве $\boldsymbol{b}$ (соответственно, $\boldsymbol{b}_{0}$ ) выполнены все условия, сформулированные в п. 2 .

Введем подмножество $\boldsymbol{b}^{0} \subset \boldsymbol{b}$ всех функций $g \in \boldsymbol{b}$, удовлетворяющих условию

$$
\lim _{n \rightarrow \infty}\left\|\chi_{[n, n+1, \cdots, \infty)} g\right\|_{\boldsymbol{b}}=0 .
$$

Иначе говоря, пространство $\boldsymbol{b}^{0}$ состоит из всех функций $g \in \boldsymbol{b}$, стремящихся при $t \rightarrow \infty$ к нулю по метрике пространства $\boldsymbol{b}$; нетрудно показать, что $\boldsymbol{b}^{0}$ является замкнутым подпространством пространства $\boldsymbol{b}$ и совпадает с замыканием по норме $\|\cdot\|_{\boldsymbol{b}}$ линейного многообразия всех финитных функций $g \in \boldsymbol{b}$. Аналогично определим пространство $\boldsymbol{b}_{0}^{0}$. Введем обозначение $Q_{22}=\mathcal{L}_{22} W_{22}$.

4. Теоремы о существовании пределов решений на бесконечности для ЛФДУП и ЛРУП. Ниже дополнительно предполагаем, что пространство $\boldsymbol{B}$ содержит все функции-константы, а подпространство $\boldsymbol{B}_{0}$ не содержит отличных от нуля констант.

Будем говорить, что вектор $z(\infty)=\underset{t \rightarrow \infty}{\boldsymbol{B}-\lim _{0}} z(t)$ есть $\boldsymbol{B}$-предел функции $z \in \boldsymbol{B}$, если разность $z-z(\infty)$ принадлежит пространству $\boldsymbol{B}_{0}$, т.е. если

$$
\lim _{s \rightarrow \infty}\left\|[z-z(\infty)]^{s}\right\|_{\boldsymbol{B}}=0 .
$$

Корректность этого определения следует из того, что если $\xi_{1}=\underset{t \rightarrow \infty}{\boldsymbol{B}-\lim } z(t)$ и $\xi_{2}=\underset{t \rightarrow \infty}{\boldsymbol{B} \text { - } \lim } z(t)$, то $\xi_{1}-\xi_{2} \in \boldsymbol{B}_{0}$. Отсюда $\xi_{1}=\xi_{2}$ ввиду свойств пространства $\boldsymbol{B}_{0}$.

Для $(n \times n)$-матрицы $A(t)=\left\{A_{1}(t), \ldots, A_{n}(t)\right\}$ будем писать

Если $\boldsymbol{B}=L_{\infty}$, то

$$
A(\infty)=\underset{t \rightarrow \infty}{\boldsymbol{B}-\lim _{i}} A(t)=\left\{\underset{t \rightarrow \infty}{\boldsymbol{B}-\lim _{1}} A_{1}(t), \ldots, \underset{t \rightarrow \infty}{\boldsymbol{B}-\lim _{n}} A_{n}(t)\right\} .
$$

$$
z(\infty)=\underset{t \rightarrow \infty}{\operatorname{vrai} \lim } z(t), \quad A(\infty)=\underset{t \rightarrow \infty}{\operatorname{vrai} \lim } A(t)
$$

Обозначим через $\boldsymbol{B}_{l}$ пространство функций $z \in \boldsymbol{B}$, для каждой из которых существует $\boldsymbol{B}$-предел с нормой $\|z\|_{\boldsymbol{B}_{l}}=\|z\|_{\boldsymbol{B}}$. Аналогично определим пространство $C_{l}$ функций $x \in C$, для которых существует $\lim _{t \rightarrow \infty} x(t)=x(\infty) \in \mathbb{R}^{n}$ с нормой $\|x\|_{C_{l}}=\|x\|_{C}$.

Относительно модельного уравнения $\mathcal{L}_{11}^{0} x=z$ и пространства $\boldsymbol{B}$ будем предполагать следующеe:

(a) оператор Коши $W_{11}$ действует из пространства $\boldsymbol{B}_{l}$ в пространство $C_{l}$ и ограничен;

(b) столбцы фундаментальной матрицы $U_{11}$ принадлежат пространству $C_{l}$.

Эти условия означают, что имеет место непрерывное вложение $\boldsymbol{D}\left(\mathcal{L}_{11}^{0}, \boldsymbol{B}_{l}\right) \subset C_{l}$. Следовательно, предполагается, что фундаментальная матрица $U_{11}$ имеет конечный предел $U_{11}(\infty)=\lim _{t \rightarrow \infty} U_{11}(t)$. Имеет место непрерывное вложение $\boldsymbol{D}\left(\mathcal{L}_{11}^{0}, \boldsymbol{B}\right) \subset C$.

Отметим, что для интегральных уравнений утверждения о наличии пределов решений были получены ранее в $[12,13,22-24]$; некоторыми идеями этих работ мы воспользовались.

Здесь предполагаем, что пространство $\boldsymbol{b}$ содержит все функции-константы, а подпространство $\boldsymbol{b}^{0}$ не содержит отличных от нуля констант. Через $\boldsymbol{b}^{l}$ обозначим пространство функций $g \in \boldsymbol{b}$, для которых существует $\boldsymbol{b}$-предел $g(\infty)$ с нормой $\|g\|_{\boldsymbol{b}^{l}}=\|g\|_{\boldsymbol{b}}$. Иначе говоря, пространство $\boldsymbol{b}^{l}$ состоит из всех функций $g \in \boldsymbol{b}$, стремящихся при $n \rightarrow \infty, n \in \mathbb{N}$, к пределу $g(\infty)$ по метрике пространства $\boldsymbol{b}$. Нетрудно показать, что $\boldsymbol{b}^{l}$ является замкнутым подпространством пространства $\boldsymbol{b}$ и совпадает с замыканием по норме $\|\cdot\|_{\boldsymbol{b}}$ линейного многообразия всех финитных функций $g \in \boldsymbol{b}$ и функций-констант. Аналогично определяем пространство $\boldsymbol{b}_{0}^{l}$.

Всюду будем предполагать, что для пространства $\boldsymbol{b}$ и модельного уравнения $\mathcal{L}_{22}^{0} y=u$ выполнены следующие условия:

(a) оператор Коши $W_{22}$ действует из пространства $\boldsymbol{b}^{l}$ в пространство $\ell_{\infty 0}^{l}$ и ограничен;

(b) столбцы фундаментальной матрицы $U_{22}$ уравнения $\mathcal{L}_{22}^{0} y=0$ принадлежат пространству $\ell_{\infty 0}^{l}$. Таким образом, имеет место непрерывное вложение $\boldsymbol{D}\left(\mathcal{L}_{22}^{0}, \boldsymbol{b}^{l}\right) \subset \ell_{\infty 0}^{l}$ и существование пределов

$$
\lim _{n \rightarrow \infty} y(n)=\lim _{n \rightarrow \infty} y[n]=y(\infty)
$$


всех решений уравнения $\mathcal{L}_{22}^{0} y=g$ в случае $g \in \boldsymbol{b}^{l}$.

Имеет место непрерывное вложение $\boldsymbol{D}\left(\mathcal{L}_{22}^{0}, \boldsymbol{b}\right) \subset \ell_{\infty 0}$.

5. Асимптотически периодические решения. Пусть $\omega$-фиксированное положительное число. Определим оператор сдвига $S^{\omega}$ равенством $\left(S^{\omega} z\right)(t)=z(t+\omega)$. Будем предполагать, что пространство $\boldsymbol{B}$ содержит все функции-константы, $S^{\omega} \boldsymbol{B} \subset \boldsymbol{B}$ и $\left\|S^{\omega}\right\|_{\boldsymbol{B} \rightarrow \boldsymbol{B}}=1$. Таким образом, пространство $\boldsymbol{B}_{0}$ не содержит отличных от нуля функций-констант. Обозначим через $\boldsymbol{B}_{\omega}$ подпространство всех асимптотически $\omega$-периодических функций: $z \in \boldsymbol{B}_{\omega}$, если $\left(S^{\omega} z-z\right) \in \boldsymbol{B}_{0}$. Аналогично определяем пространство $C_{\omega}$, элементами которого являются такие $x \in C$, что $\left(S^{\omega} x-x\right) \in C_{0}$. Ниже будем предполагать, что пространство $\boldsymbol{B}$ вольтеррово допустимо для модельного уравнения $\mathcal{L}_{11}^{0} x=z$ и пространство $D\left(\mathcal{L}_{11}^{0}, \boldsymbol{B}_{\omega}\right)$ вложено в пространство $C_{\omega}$. Таким образом, $\boldsymbol{D}\left(\mathcal{L}_{11}^{0}, \boldsymbol{B}_{\omega}\right)$-устойчивость уравнения $\mathcal{L}_{11} x=f$ означает, что если $f \in \boldsymbol{B}_{\omega}$, то любое решение $x$ этого уравнения является асимптотической $\omega$-периодической функцией.

Введем обозначение $\left[S^{\omega}, Q_{11}\right]=S^{\omega} Q_{11}-Q_{11} S^{\omega}$, где $Q_{11}=\mathcal{L}_{11} W_{11}$. Пусть модельное уравнение $\mathcal{L}_{11}^{0} x=z$ удовлетворяет условиям (а) и (b) из п. 4.

Теорема 1. Пусть уравнение $\mathcal{L}_{11} x=f$ является $\boldsymbol{D}\left(\mathcal{L}_{11}^{0}, \boldsymbol{B}\right)$-устойчивым, а оператор

$$
\mathcal{L}_{11}: \boldsymbol{D}\left(\mathcal{L}_{11}\right) \rightarrow L
$$

действует из пространства $\boldsymbol{D}\left(\mathcal{L}_{11}^{0}, \boldsymbol{B}_{l}\right)$ в пространство $\boldsymbol{B}_{l}$ и ограничен, а такэе

$$
\left[S^{\omega}, \mathcal{L}_{11} W_{11}\right] \boldsymbol{B} \subset \boldsymbol{B}_{l} .
$$

Тогда это уравнение $D\left(\mathcal{L}_{11}^{0}, \boldsymbol{B}_{\omega}\right)$-устойчиво.

Эти утверждения дополняют результаты работ $[7,8]$.

Лемма 1. Пусть линейный ограниченный оператор $Q_{11}: \boldsymbol{B} \rightarrow \boldsymbol{B}$ вольтерров, $Q_{11} \boldsymbol{B} \subset \boldsymbol{B}$. Далее, пусть оператор $\left[S^{\omega}, Q_{11}\right]$ переводит пространство $\boldsymbol{B}$ в пространство $\boldsymbol{B}_{l}$. Тогда оператор $Q_{11}$ действует в пространстве $\boldsymbol{B}_{\omega}$. Если, кроме того, оператор $Q_{11}: \boldsymbol{B} \rightarrow \boldsymbol{B}$ имеет обратный оператор $Q_{11}^{-1}: \boldsymbol{B} \rightarrow \boldsymbol{B}$, то оператор $Q_{11}^{-1}$ действует в пространстве $\boldsymbol{B}_{\omega}$.

Теорема 1 и лемма 1 опубликованы в статье [3] и в книге [4][гл. IV, § 4.4, теорема 4.4.1 и лемма 4.4.2]. Отметим, что для интегральных уравнений Вольтерра утверждения, аналогичные лемме 1, приведены в обзоре [22][гл. 2, § 2].

Пусть $\omega \in \mathbb{N}$ - фиксированное число. Будем предполагать, что пространство $\boldsymbol{b}$ содержит все функции-константы, $S^{\omega} \boldsymbol{b} \subset \boldsymbol{b}$ и $\left\|S^{\omega}\right\|_{\boldsymbol{b} \rightarrow \boldsymbol{b}}=1$. Таким образом, пространство $\boldsymbol{b}^{0}$ не содержит отличных от нуля функций-констант. Обозначим через $\boldsymbol{b}^{\omega}$ подпространство всех асимптотически $\omega$-периодических функций: $u \in \boldsymbol{b}^{\omega}$, если $\left(S^{\omega} u-u\right) \in \boldsymbol{b}^{0}$. Аналогично определяем пространство $\ell_{\infty 0}^{\omega}$, элементами которого являются такие функции $y \in \ell_{\infty 0}$, что $\left(S^{\omega} y-y\right) \in \ell_{\infty 0}^{0}$. Ниже будем предполагать, что пространство $\boldsymbol{b}$ вольтеррово допустимо для модельного уравнения $\mathcal{L}_{22}^{0} g=u$ и пространство $\boldsymbol{D}\left(\mathcal{L}_{22}^{0}, \boldsymbol{b}^{\omega}\right)$ вложено в пространство $\ell_{\infty 0}^{\omega}$. Таким образом, $\boldsymbol{D}\left(\mathcal{L}_{22}^{0}, \boldsymbol{b}^{\omega}\right)$-устойчивость уравнения $\mathcal{L}_{22} y=g$ означает, что если $g \in \boldsymbol{b}^{\omega}$, то любое решение $y$ этого уравнения является асимптотической $\omega$-периодической функцией.

Введем обозначение $\left[S^{\omega}, Q_{22}\right]=S^{\omega} Q_{22}-Q_{22} S^{\omega}$, где $Q_{22}=\mathcal{L}_{22} W_{22}$. Пусть модельное уравнение $\mathcal{L}_{22}^{0} g=u$ удовлетворяет условиям (а) и (b) из п. 4.

Теорема 2. Пусть уравнение $\mathcal{L}_{22} y=$ g является $\boldsymbol{D}\left(\mathcal{L}_{22}^{0}, \boldsymbol{b}\right)$-устойчивым, а оператор

$$
\mathcal{L}_{22}: \boldsymbol{D}\left(\mathcal{L}_{22}\right) \rightarrow \ell
$$

действует из пространства $\boldsymbol{D}\left(\mathcal{L}_{22}^{l}, \boldsymbol{b}^{l}\right)$ в пространство $\boldsymbol{b}^{l}$ и ограничен, а также

$$
\left[S^{\omega}, \mathcal{L}_{22} W_{22}\right] \boldsymbol{b} \subset \boldsymbol{b}^{l} .
$$

Тогда это уравнение $\boldsymbol{D}\left(\mathcal{L}_{22}^{0}, \boldsymbol{b}^{\omega}\right)$-устойчиво.

Лемма 2. Пусть линейный ограниченный оператор $Q_{22}: \boldsymbol{b} \rightarrow \boldsymbol{b}$ вольтерров, $Q_{22} \boldsymbol{b} \subset \boldsymbol{b}$. Пусть, далее, оператор $\left[S^{\omega}, Q_{22}\right]$ переводит пространство $\boldsymbol{b}$ в пространство $\boldsymbol{b}^{l}$. Тогда оператор $Q_{22}$ действует в пространстве $\boldsymbol{b}^{\omega}$. Если, кроме того, оператор $Q_{22}: \boldsymbol{b} \rightarrow \boldsymbol{b}$ имеет обратный оператор $Q_{22}^{-1}: \boldsymbol{b} \rightarrow \boldsymbol{b}$, mо оператор $Q_{22}^{-1}$ действует в пространстве $\boldsymbol{b}^{\omega}$. 
Сформулируем распространение теоремы Боля-Перрона на уравнение $\mathcal{L}\{x, y\}=\{f, g\}$.

Теорема 3. Пусть $\omega \in \mathbb{N}$ - фиксированное число. Предположим, что выполнены следующие условия: операторы

$$
\mathcal{L}_{12}: \boldsymbol{D}\left(\mathcal{L}_{22}^{0}, \boldsymbol{b}\right) \rightarrow \boldsymbol{B}, \quad \mathcal{L}_{21}: \boldsymbol{D}\left(\mathcal{L}_{11}^{0}, \boldsymbol{B}\right) \rightarrow \boldsymbol{b}
$$

действуют и ограничен, причем

$$
\mathcal{L}_{12}\left(\boldsymbol{D}\left(\mathcal{L}_{22}^{0}, \boldsymbol{b}^{l}\right)\right) \subset \boldsymbol{B}_{l}, \quad \mathcal{L}_{21}\left(\boldsymbol{D}\left(\mathcal{L}_{11}^{0}, \boldsymbol{B}_{l}\right)\right) \subset \boldsymbol{b}^{l} ;
$$

onераторы

$$
\mathcal{L}_{11}: \boldsymbol{D}\left(\mathcal{L}_{11}\right) \rightarrow L, \quad \mathcal{L}_{22}: \boldsymbol{D}\left(\mathcal{L}_{22}\right) \rightarrow \ell
$$

непреръвно действуют из пространств $\boldsymbol{D}\left(\mathcal{L}_{11}^{0}, \boldsymbol{B}_{l}\right)$ и $\boldsymbol{D}\left(\mathcal{L}_{22}^{0}, \boldsymbol{b}^{l}\right)$ в пространства $\boldsymbol{B}_{l}$ и $\boldsymbol{b}^{l}$ и справедливы включения

$$
\left[S^{\omega}, \mathcal{L}_{11} W_{11}\right] \boldsymbol{B} \subset \boldsymbol{B}_{l}, \quad\left[S^{\omega}, \mathcal{L}_{22} W_{22}\right] \boldsymbol{b} \subset \boldsymbol{b}^{l} .
$$

Пусть уравнение $\mathcal{L}_{11} x=f$ является $\boldsymbol{D}\left(\mathcal{L}_{11}^{0}, \boldsymbol{B}\right)$-устойчивым, а и уравнение $\mathcal{L}_{22} y=g-\boldsymbol{D}\left(\mathcal{L}_{22}^{0}, \boldsymbol{b}\right)$ устойчивым. Далее, пусть уравнение

$$
\mathcal{L}_{1} x=\left(\mathcal{L}_{11}-\mathcal{L}_{12} \mathcal{C}_{22} \mathcal{L}_{21}\right) x=f_{1}
$$

является $\boldsymbol{D}\left(\mathcal{L}_{11}^{0}, \boldsymbol{B}\right)$-устойчивым, оператор $\mathcal{L}_{1} W_{11}: \boldsymbol{B}_{l} \rightarrow \boldsymbol{B}_{l}$ ограничен и справедливо включение

$$
\left[S^{\omega}, \mathcal{L}_{1} W_{11}\right] \boldsymbol{B} \subset \boldsymbol{B}_{l} .
$$

Тогда уравнение $\mathcal{L}\{x, y\}=\operatorname{col}\{f, g\}$ будет $\boldsymbol{D}\left(\mathcal{L}_{0}, \boldsymbol{B}_{\omega} \times \boldsymbol{b}^{\omega}\right)$-устойчивым.

Предложенная статья продолжает исследование, начатое в [14-20,24]. В [14-16, 24] получена теорема Боля-Перрона для ГЛСФДУП в случае экспоненциальной устойчивости. В $[17,18]$ получена теорема Боля-Перрона для ГЛСФДУП в случае асимптотической устойчивости. В [19] получено обращение к теореме Боля-Перрона для ГЛСФДУП в случае асимптотической устойчивости. В [20] получена теорема Боля-Перрона об асимптотических периодических решениях для ГЛСФДУП в случае асимптотической устойчивости.

\section{СПИСОК ЛИТЕРАТУРЫ}

1. Азбелев Н. В., Березанский Л. М., Симонов П. М., Чистяков А. В. Устойчивость линейных систем с последействием. II// Диффер. уравн. - 1991. - 27, № 4. - С. 555-562.

2. Азбелев Н. В., Березанский Л. М., Симонов П. М., Чистлков А. В. Устойчивость линейных систем с последействием. III// Диффер. уравн. - 1991. - 27, № 10. - С. 1659-1668.

3. Азбелев Н. В., Березанский Л. М., Симонов П. М., Чистлков А. В. Устойчивость линейных систем с последействием. IV// Диффер. уравн. - 1993. - 29, № 2. - С. 196-204.

4. Азбелев Н. В., Симонов П. М. Устойчивость решений уравнений с обыкновенными производными. Пермь: Перм. ун-т, 2001.

5. Барбашин Е. А. Введение в теорию устойчивости. - М.: Наука, 1967.

6. Далецкий Ю. Л., Крейн М. Г. Устойчивость решений дифференциальных уравнений в банаховом пространстве. - М.: Наука, 1970.

7. Домошницкий А. И., Драхлин М. Е. О периодических решениях функционально-дифференциальных уравнений// Докл. семин. Ин-та прикл. мат. им. И. Н. Векуа - 1988. - 3, № 3. - С. 54-57.

8. Драхлин M. E. Один признак существования асимптотически периодических решений// в кн.: Функцтонально-дифференциальные уравнения/ Межвуз. сб. науч. тр.. - Пермь: Перм. политехн. ин-т, 1990. - С. 168-170.

9. Канторович Л. В., Акилов Г. П. Функциональный анализ. - СПб.: Невский Диалект, 2004.

10. Крейн М. Г. О некоторых вопросах, связанных с кругом идей Ляпунова в теории устойчивости// Усп. мат. наук. - 1948. - 3, № 3 (25). - С. 166-169.

11. Массера X. Л., Шеффер X. Х. Линейные дифференциальные уравнения и функциональные пространства. - М.: Мир, 1970.

12. Пуляев $B . \Phi$. О допустимости некоторых пар пространств относительно линейных интегральных уравнений Вольтерра// Диффер. уравн. - 1984. - 20, № 10. - С. 1800-1805. 
13. Пуляев В. Ф. О спектре линейных непрерывных операторов// Изв. Сев.-Кав. науч. центра высш. школы. Естеств. науки. - 1985. - № 4. - С. 25-28.

14. Симонов П. М. Теорема Боля-Перрона для гибридных линейных систем с последействием// Вестн. Перм. ун-та. Мат. Мех. Информ. - 2016. - № 2 (33). - С. 56-60.

15. Симонов П. М. К вопросу о теореме Боля-Перрона для гибридных линейных функциональнодифференциальных систем с последействием// Ж. Средневолж. мат. о-ва. - 2016. - 18, № 1 . C. $75-81$.

16. Симонов П. М. Теорема Боля-Перрона для гибридных линейных систем с последействием// Итоги науки техн. Совр. мат. прилож. Темат. обзоры. - 2017. - 132. - С. 122-126.

17. Симонов П. М. Теорема Боля-Перрона об асимптотической устойчивости для гибридных линейных функционально-дифференциальных систем с последействием// Вестн. РАЕН. Диффер. уравн. - 2016. - 16, № 3. - С. 55-59.

18. Симонов П. М. Теорема Боля-Перрона об асимптотической устойчивости гибридных систем// в кн.: Функционально-дифференциальные уравнения: теория и приложения/ Мат. конф., посв. 95летию со дня рожд. проф. Н. В. Азбелева (Пермь, 17-19 мая 2017 г.). - Пермь, 2018. - С. 230-235.

19. Симонов П. М. Теорема Боля-Перрона и обратная к ней об асимтотической устойчивости для гибридных линейных систем с последействием// Вестн. Перм. ун-та. Мат. Мех. Информ. - 2018. - 2, № 41. - C. 38-43.

20. Симонов П. М. Теорема Боля-Перрона об асимптотически периодических решениях для гибридных линейных функционально-дифференциальных систем с последействием// Вестн. РАЕН. Диффер. уравн. - 2018. - 18, № 4. - С. 58-64.

21. Сокол Д. Г. О допустимости некоторых пар пространств для интегральных операторов и уравнений// Изв. вузов. Сев.-Кав. рег. Естеств. науки. - 2000. - 1. - С. 135-137.

22. Цалюк 3. Б. Интегральные уравнения Вольтерра// в кн.: Итоги науки и техн. Мат. анализ. - М.: ВИНИТИ, 1977. - 15. - С. 131-198.

23. Цалюк 3. Б. О допустимости некоторых пар пространств для интегральных операторов и уравнений Вольтерра// Диффер. уравн. - 1977. - 13, № 11. - С. 2096-2098.

24. Simonov P. M. The Bohl-Perron theorem for hybrid linear systems with aftereffect// J. Math. Sci. — 2018. - 230, № 5. - P. 775-781.

Симонов Петр Михайлович

Пермский государственный национальный исследовательский университет

E-mail: simpm@mail.ru 